\title{
The Gut Microbiota and Pediatric Multiple Sclerosis: Recent Findings
}

\author{
Helen Tremlett ${ }^{1}$ • Emmanuelle Waubant ${ }^{2}$
}

Published online: 22 September 2017

(C) The American Society for Experimental NeuroTherapeutics, Inc. 2017

\begin{abstract}
Pediatric multiple sclerosis (MS) is a chronic, lifelong neurological condition associated with inflammation and degeneration in the brain and spinal cord. Fortunately, $<5 \%$ of people with MS have their onset in childhood years. However, studying these very-early-onset cases of MS offers key advantages. In particular, with fewer years lived, children have had a limited range of exposures, potentially enhancing our ability to identify what might cause MS. Further, as the actual timing of the biological MS onset is unknown, the possibility to study these children much closer to the real onset of disease is far greater than in the typical adult with MS. Whether MS (in children or adults) can be prevented is unknown and the available drugs are only modestly effective in modifying the disease course and are not without risk. Emerging evidence is providing insight into the gut microbiota's potential role in triggering and shaping neurological conditions such as MS. The limited number of studies in humans with MS and absence of prior work in pediatric MS motivated the following 3 fundamental questions, addressed in 2 cross-sectional and 1 longitudinal investigation in children with and without MS: 1) Does the gut microbiota composition differ between children with and without MS? 2) Is there an association between the gut microbiota and host immune markers in children with and without MS? 3) Is the gut microbiota associated with the future risk of a MS relapse?
\end{abstract}

Helen Tremlett

helen.tremlett@ubc.ca

1 Medicine (Neurology) and the Djavad Mowafaghian Centre for Brain Health, University of British Columbia, Vancouver, BC, Canada

2 University of California, San Francisco, San Francisco, CA, USA
Keywords Multiple sclerosis $\cdot$ Pediatric $\cdot$ Microbiome $\cdot$ Microbiota $\cdot$ Relapsing-remitting $\cdot$ Disease-modifying drugs . Case-control · Cross-sectional $\cdot$ Longitudinal $\cdot$ Relapse risk · 16S rRNA $\cdot$ Metagenomics

\section{Introduction}

Multiple sclerosis (MS) is considered an immune-mediated disease, with several genetic and early-life environmental exposures implicated as potential triggers for both adult- and childhood-onset disease [1-5]. However, there is no clinically useful test to predict who might be at risk of developing MS and no proven means of modifying an individual's risk to prevent the onset of MS. Once MS develops, the disease course can be highly variable between individuals [6], and very little is known as to what drives this heterogeneity [5, 6]. Recent work, primarily based on animal models of MS, suggests a role for the gut microbiota as both a trigger of disease onset and as a modifier of subsequent disease activity $[7,8]$. Perturbation in the gut microbiota of adults with MS relative to controls without MS have also been reported [9-14]. While of interest, many of these studies included adults with long-standing MS who typically have complex medical and lifestyle histories. This can make the potential search for disease trigger(s) or modifiers particularly challenging as some may have occurred years previously [15] or accumulated over decades. Pediatric MS, while relatively rare, represents a unique opportunity to examine disease processes after few years of life have accrued, narrowing the search for potential triggers or modifiers of disease [1-4]. Evidence to date also suggests that findings observed in children with MS are generalizable to adults (and vice versa). For instance, of the main risk factors identified for MS onset to date (low serum vitamin D, exposure to Epstein-Barr virus, and high 
body mass index), best evidence implicates them in both pediatric and adult-onset MS [1-5]. This suggests that the 2 populations represent similar disease entities, making it reasonable to assume that aspects of the gut microbiota-related findings in pediatric MS are generalizable to the adult MS population (and vice versa). However, while pediatric MS represents a unique opportunity to explore the role of the gut microbiota as a modifier of disease processes, its relatively low incidence and prevalence presents challenges. Gaining access to sufficient numbers of new cases of pediatric MS requires well-coordinated, collaborative teams and networks. The findings summarized in this article relate to children with and without MS attending a MS or "wellness" pediatric clinic at the University of California, San Francisco (UCSF), USA, part of the US Network of Pediatric MS Centers [16-18]. All the MS cases were enrolled very early in their disease course, with stool samples obtained within 2 years of MS symptom onset. Findings provide an intriguing insight into the potential role of the gut microbiota in this chronic neurological condition and set the scene for future investigations.

\section{The Gut Microbiota in Pediatric MS: Preliminary Findings}

\section{Does the Gut Microbiota Differ Between Children With and Without MS?}

At the time this question was posed, the primary available evidence implicating the gut microbiota in MS stemmed from animal models, such as experimental autoimmune encephalomyelitis [7, 8]. Only humans are known to spontaneously develop MS. Other animals require a noxious cocktail of chemicals in order to develop a disease that mimics certain facets of the relapsing-remitting MS disease course. Nonetheless, these animal models provided a compelling rationale to extend studies to humans. In the face of many unknowns, and no other published studies in MS, a case-control study was initiated in order to address the fundamental questions of whether there were any measurable differences in the gut microbiota profiles of children with and without MS [16]. Several potential confounders of the relationship between the gut microbiota and MS were also collected in order to guide future studies. These included (but were not limited to) environmental and lifestyle factors, such as current and early life diet (including breastfeeding), mode of delivery, body mass index, medication history, animal exposures, and MS-specific clinical factors, such as relapse history and disability status [16]. This level of deep phenotyping is important in order to contextualize and interpret findings, as well as compare and contrast across other studies, both now and in the future.

Between 2011 and 2013, 18 children with disease onset before the age of 18 years attending the UCSF pediatric MS clinic were enrolled. Cases were within 24 months of MS symptom onset and all met the 2010 McDonald criteria for MS [19]. Seventeen age- and sex-similar control children were enrolled; all had no personal or family history of MS or a related autoimmune disease, although conditions such as asthma or eczema were permissible. No child had been exposed to a systemic antibiotic in the 2 months prior to stool sample collection. Stool samples were procured at home and mailed by the child's parent along with the provided icepack (to keep the samples cool but avoiding repeated freezethawing and associated microbial degradation) [20]. Stool samples were then stored at $-80^{\circ} \mathrm{C}$ at the study laboratory prior to DNA extraction, 16S rRNA gene amplification, and related sequencing using the Illumina Miseq platform. Additional details have been previously published, including the complementary chip-based platform, which was applied to provide a means of validating findings (and which yielded similar results; not reported here) [16].

In this cohort of children, the mean age was 13 years (range 4-18 years), just over half were girls $(19 / 34 ; 56 \%)$ and the MS disease duration was short, averaging 11 months (range 2-24 months). Half of the cases were not yet exposed to an immunomodulatory drug (IMD) for MS. Overall, the diversity of the gut microbiota did not differ significantly between children with and without MS for either alpha (evenness, richness, and Faith's phylogenic metric) or beta diversity (Canberra distance matrix). These findings are comparable with those observed in adults; generally, gut diversity (alpha and/or beta) has been reported as rather similar between adults with MS and unaffected controls [10-12, 14]. The issue of IMD exposure and gut diversity is addressed later in this review article.

In contrast to the general lack of difference in gut diversity between MS cases and controls, we did observe differences between children with and without MS when the gut microbiota were assessed at lower taxonomic levels [16]. This again mirrors the situation in adults in which taxonomic differences have been reported in adults with MS relative to controls $[10-12,14]$.

Starting at the broad phylum level (Fig. 1), the children with MS in our study had 2.5 times the relative abundance of Actinobacteria relative to controls [95\% confidence interval (CI) 1.3-4.9), based on negative binomial regression [16]. Overabundance of this phylum has also been associated with other relapsing-remitting immune-mediated conditions such as inflammatory bowel disease [21]. At lower taxonomic rankings, the MS cases (relative to controls) had a higher relative abundance of the genera Desulfovibrio and Bifidobacterium and the Christensenellaceae family, as well as decreased relative abundance of the Clostridiales order (e.g., the butyrate-producing Lachnospiraceae and Ruminococcaceae). The association of MS with the reportedly highly heritable taxon Christensenellaceae [22] is of interest given that MS has known (host) familial and genetic 


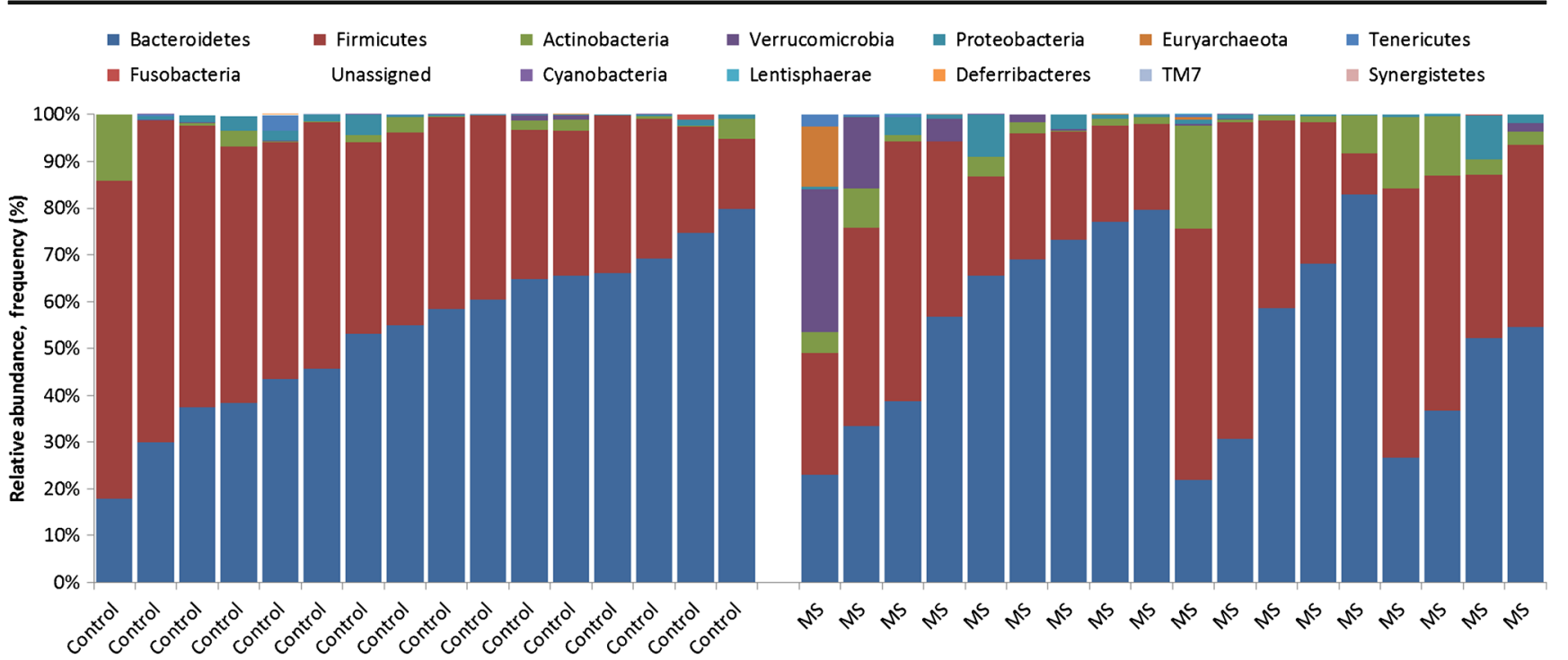

Fig. 1. Relative abundance of the gut microbiota phyla for cases and controls using 16S rRNA sequencing [16]. Each bar represents 1 child; controls (left) and multiple sclerosis cases (right). The $y$-axis depicts relative abundance of the gut microbiota phyla, reported as a proportion for

each child, relative to all cases and controls. Each phyla are color-coded as shown; e.g., as expected the predominant phyla in the guts of all children (cases and controls) with Bacteroidetes (blue) and Firmicutes (red)

contributions [23]. In addition, recent work has suggested a relationship between the mode of delivery and MS risk, with a Cesarian section associated with lower odds of developing pediatric MS (adjusted odds ratio 0.40 ; 95\% CI 0.20-0.82; $p=0.01$ ) [24]. Desulfovibrio have been found in other studies to be associated with inflammatory bowel disease [25] and, specifically, ulcerative colitis [26]. Similarly, increased abundance of Bifidobacterium (phylum Actinobacteria) have been observed in immune-mediated inflammatory diseases, specifically in children and adults with celiac disease $[27,28]$ and adults with Crohn's disease [29] and ulcerative colitis [30].

Combined, findings indicate that even in the very early stages of pediatric MS, subtle differences can be observed relative to control children. These findings are indicative of a higher proinflammatory and lower anti-inflammatory gut microbiota milieu in the children with MS [30,31]. While these observations were largely consistent, regardless of the IMD exposure status of the MS cases (typically glatiramer acetate or interferon- $\beta$ was used), IMD exposure did influence other observations. In particular, beta diversity was associated with IMD exposure (Canberra, $p<0.02$ ), an observation consistent with that found in adults with MS also taking glatiramer acetate [10]. A plausible explanation is that IMD exposure caused these differences, but patient characteristics, such as the underlying disease activity, can also influence the decision to initiate an IMD and may have contributed. Longitudinal studies are needed to elucidate these crosssectional associations [16].

With the goal of gaining insight into the potential function of the gut microbiota, a validated algorithm was applied to the 16S rRNA sequencing data ["Phylogenetic Reconstruction of Unobserved States" (PICRUSt)] [32]. This allows inferences

to be made regarding the metagenome and hence potential functionality of the gut microbiota. A predicted enrichment of microbial genes involved in glutathione metabolism was observed in the MS cases relative to controls (MannWhitney, $p=0.017$ ), which was independent of IMD exposure [16]. Glutathione has been implicated in MS neurodegeneration, and pursued as a therapeutic target or possible (brain) biomarker [33]. The potential for glutathione involvement beyond the confines of the central nervous system, possibly via the gut microbiota's interactions with metabotropic glutamate gut receptors, might provide opportunity and merits future investigation [34]. The IMD exposure status of the MS children influenced some findings, such as the predicted enrichment of microbial genes encoding immunomodulatory pathways involving $\mathrm{CD} 8^{+}, \mathrm{CD}^{+}$, and B-cell maturation and differentiation [16]. Reassuringly, these observations are consistent with the known pharmacodynamics of the IMDs [35]. Moving forward, understanding this functional capacity will be invaluable if, for instance, future interventions, such as a drug, specific diet, or pre-/probiotics, are developed in an attempt to "normalize" the MS gut.

\section{Summary}

Subtle differences in the gut microbiota were observed in pediatric MS relative to control children [16]. Similar observations made in adults with MS [9] raise the possibility of a gut signature of MS [36]. However, exposure to an IMD was associated with greater differences in the gut microbiota composition than MS itself [16]. Further, there was a suggestion of some commonality with certain other chronic, relapsing conditions [16]. It will be interesting for future studies to ascertain 
which aspects are entirely unique to MS, which are influenced by exposure to drug (and/or perhaps other exposures), and which represent an overlapping spectrum with other immune-mediated inflammatory conditions.

\section{Is There an Association Between the Gut Microbiota and Host Immune Markers in Children With and Without MS?}

The gut microbiota play a key role in educating and modifying the host's immune response. In turn, the host's immune system monitors the activity of the resident and transient gut microbes [37, 38]. As shifts in the immune profiles of individuals with MS are considered a key aspect of the disease [39, 40], and studies have now implicated involvement of the gut microbiota in MS, it is of relevance to understand the evolving "microbiota-immune" relationship. However, very little is currently known. To help fill this knowledge gap, we set out to examine the association between the gut microbiota and host immune system in children with and without MS [18]. Individuals were enrolled from the UCSF's pediatric clinics, as outlined previously $[16,18]$. A subset of children (15 MS cases, 9 controls) who contributed both a stool and blood sample were included. Details of the blood sample processing and immune profiling have been previously published [18]. Briefly, peripheral blood mononuclear cells were isolated and stimulated, and, using flow cytometry, regulatory $\mathrm{T}$ cell (Treg; $\mathrm{CD} 4{ }^{+} \mathrm{CD} 25^{\text {hi }} \mathrm{CD} 127^{\text {low }} \mathrm{FoxP}^{+}$) frequency and $\mathrm{CD} 4^{+} \mathrm{T}$-cell intracellular cytokine production was evaluated [18]. Associations between microbiota diversity, phylum-level abundances, and immune markers were explored, initially using Pearson's correlation coefficient (r), followed by multivariate linear regression, adjusting for potential confounders. Potential confounders included age, disease duration (both as continuous variables), or IMD exposure status (exposed or naïve). These were selected based on clinical relevance and/ or the limited pediatric literature, which indicated that these factors could influence the relationship between the developing gut microbiota and immune system [16, 17]. When immune markers were compared between cases and controls, most [e.g., T helper (Th)2, Th17, Tregs] did not differ significantly between groups $(p>0.05)$. However, divergence in the relationship between the gut microbiota and immune markers were observed [18]. Some striking examples included the strong, inverse correlations between gut microbiota evenness (indicating taxon distribution and a measure of gut microbial diversity) and Th17 and Th2 blood makers for the control children $(\mathrm{r}=-0.728$ and $\mathrm{r}=-0.750$, respectively; both $p<$ $0.05)$. However, there was either no or modest positive associations for the MS cases $(r=0.308$ and $\mathrm{r}=0.545$, respectively; both $p>0.05$ ). After model adjustments, both immune markers remained significantly associated with evenness for the controls (age-adjusted $p<0.05$ ). Findings suggest that gut microbiota dominated by specific taxa were associated with decreases in these immune markers in control children but that this effect was lost or absent in the cases [18].

At the phylum level (Table 1), the relative abundance of Bacteroidetes, one of the most dominant phyla in the gut, inversely associated with Th17 for cases $(\mathrm{r}=-0.719, p=$ $0.008)$ but not controls ( $\mathrm{r}=0.320, p=0.401)$. This was independent of IMD exposure and disease duration (linear regression, $p<0.025$ for cases). In addition, Fusobacteria positively correlated with Tregs for controls $(\mathrm{r}=0.829, p=0.006)$ but not cases $(\mathrm{r}=-0.069, p=0.808)$. This remained independent of age (adjusted linear regression, $p=0.009$ for controls). These observations concur with mechanistic studies involving animal models of MS [41]. Members of the Bacteroidetes phylum, which includes Bacteroides fragilis, have been shown to modulate the immune system and to modify disease outcomes in murine models, likely via the gut microbiota [41-43]. Although less is known about Fusobacteria, this diverse phylum includes anaerobic, Gram-negative bacteria, similar to Bacteroidetes, raising the possibility that a similar pathway is involved.

\section{Summary}

Findings suggest that the relationship between the gut microbiota composition and host blood immune markers can differ between children with and without MS. Why this microbiotaimmune balance appears disrupted so early in the MS disease course is intriguing and motivates further work. Identification of key microbiota members involved and the mechanism(s) by which this occurs (e.g., via gut microbiota metabolites, such as the short-chain fatty acid, butyrate [37, 38]) could pave the way to novel means of modulating the immune dysregulation in MS.

\section{Is the Gut Microbiota Associated With Future Relapse Risk?}

Surprisingly little is known as what triggers or facilitates an MS relapse [5]. However, alterations of the gut microbiota in animal models of MS have been associated with disease activity [7, 8]. In addition, involvement of the immune system in MS relapses has been shown [39]. It is highly feasible that one of the key modulators of the immune system - the gut microbiota - could play a role in MS relapses. This study aimed to assess the association between the gut microbiota and subsequent relapse risk in pediatric MS [17]. The study setting and stool collection were similar to before [16], with MS cases enrolled through a UCSF pediatric MS clinic. All were within 2 years from MS symptom onset at stool sample collection (mean $=10.3$ months). This represented study entry or "baseline", with children followed prospectively for a mean of 19.8 months during which time relapse-related data were collected; all relapses were confirmed 
Table 1. Associations between the relative abundance of gut microbiota phyla and blood immune markers in cases of pediatric multiple sclerosis (MS) and control children [18]

\begin{tabular}{|l|l|l|l|l|l|}
\hline $\begin{array}{l}\text { Pearson's } \\
\text { correlation }(\mathrm{r}) \\
{ }^{*} \mathrm{p}<0.05^{* *} \mathrm{p}<0.01\end{array}$ & $\begin{array}{l}\text { All } \\
\text { children } \\
(\mathrm{n}=24)\end{array}$ & $\begin{array}{l}\text { Controls } \\
\text { only }(\mathrm{n}=9)\end{array}$ & $\begin{array}{l}\text { MS cases } \\
\text { only }(\mathrm{n}=15)\end{array}$ & $\begin{array}{l}\text { MS DMD } \\
\text { naïve } \\
(n=8)\end{array}$ & $\begin{array}{l}\text { MS DMD } \\
\text { exposed } \\
(n=7)\end{array}$ \\
\hline Bacteroidetes & & & & & \\
\hline Tregs & $\mathrm{r}=-0.612^{* *}$ & $\mathrm{r}=-0.536$ & $\mathrm{r}=-0.684^{* *}$ & $\mathrm{r}=-0.628$ & $\mathrm{r}=-0.701$ \\
\hline Th17 & $\mathrm{r}=-0.350$ & $\mathrm{r}=0.320$ & $\mathrm{r}=-0.719^{* *}$ & $\mathrm{r}=-0.873^{*}$ & $\mathrm{r}=-0.569$ \\
\hline Firmicutes & & & & & \\
\hline Th17 & $\mathrm{r}=0.218$ & $\mathrm{r}=-0.384$ & $\mathrm{r}=0.724^{* *}$ & $\mathrm{r}=0.260$ & $\mathrm{r}=0.723$ \\
\hline Th2 & $\mathrm{r}=0.065$ & $\mathrm{r}=-0.824^{*}$ & $\mathrm{r}=0.429$ & $\mathrm{r}=-0.713$ & $\mathrm{r}=0.269$ \\
\hline Fusobacteria & & & & & \\
\hline Tregs & $\mathrm{r}=-0.015$ & $\mathrm{r}=0.829^{* *}$ & $\mathrm{r}=-0.069$ & $\mathrm{r}=-0.058$ & $\mathrm{r}=-0.193$ \\
\hline
\end{tabular}

\section{Key - colour coding represents degree and direction of association}

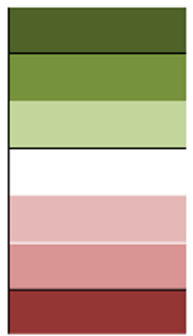

\section{Positive association}

\section{Negative association}

Select findings only shown; further details have been published elsewhere [18]

DMD disease-modifying drugs (alternative name, used interchangeably: IMD; immunomodulatory drugs)

$* p<0.05 ; * * p<0.01$

by a neurologist. Just over half ( $n=10 ; 59 \%)$ of the MS cases were girls, and nearly half self-identified as white $(n=8 ; 47 \%)$. At baseline, the mean age was 12.5 years and $8(47 \%)$ were IMD naïve. The baseline gut microbiota composition (phylum level relative abundance) was modelled as the exposure and categorized according to the data distribution as either "low vs high" ( $\leq$ $v s>$ median) or "absent $v s$ present" when detectable in $>90 \%$ of cases. Phyla with sparse data were excluded (i.e., $<20 \%$ of cases had detectable reads). The outcome was the first "on study" MS relapse [17]. "Time-to-event" survival analyses were used, which allows all individuals to be considered in the analyses, regardless if the outcome (a relapse) occurs by the study end or not. Relapse-free cases were censored at their last clinic visit. Data were assessed in 3 conservative steps, which included model adjustments in step 3. Firstly, Kaplan-Meier curves indicated that the absence of Fusobacteria (log-rank test, $p=0.001$ ), or a higher abundance of Firmicutes ( $p=0.003$ ) or the presence of the Archaea Euryarchaeota $(p=0.037)$ were each associated with a higher on study relapse risk [17]. Secondly, after applying a conservative Bonferroni correction for multiple comparisons, only the former 2 remained significant $(p<0.00625)$ and were entered into the final step. Thirdly, after covariate adjustment for age $(\leq 12$ vs $>12$ years) and IMD exposure status (exposed or naïve) using the multivariable Cox model, only Fusobacteria remained significant, with its absence ( $v s$ presence) associated with a hazard ratio for relapse risk of $3.2(95 \%$ CI $1.2-9.0 ; p=$ 0.024) [17]. Potential covariates (confounders) were selected from the baseline cohort characteristics and guided by the limited available literature, which suggested that both age and IMD exposure could be associated with both the exposure (gut microbiota) and outcome (relapse). The variables were categorized based on the data distribution. It should be noted that it is not possible to tell if there was an active decline in Fusobacteria or if overgrowth of another microbial community resulted in the observed relative depletion of Fusobacteria. Interestingly, the Fusobacteria phylum was also reported in our previous paper for its strong correlation with blood immune markers in control children but not MS cases [16, 18]. Understanding the relationship between the gut microbiota, immune markers, and relapse risk represents an important future line of inquiry.

\section{Summary}

Absence or depletion of Fusobacteria was associated with $>3$ times the hazard of an earlier relapse (adjusted for age and use of a MS IMD). A better understanding of gut microbiota's role in modifying MS relapse risk and this relationship with the 
immune system could aid identification of novel modifiable targets and improved outcomes in MS [17].

\section{Study Strengths and Challenges}

Study strengths of the 3 studies summarized here included a well-phenotyped cohort of children with and without MS [16-18]. In addition, the gut microbiota-related sequencing and related bioinformatics were conducted using a validated pipeline in a laboratory dedicated to such methods [16-18]. Case-control studies are particularly susceptible to bias. We applied the key principles of optimal study design [44], enrolling both cases and controls from the same source ("at risk") population. All children included in our 2 case-control studies were from the same geographical location and healthcare setting, assessed over a similar time period [16, 18]. We purposely did not select household controls (e.g., siblings), because of the potential for bias, as demonstrated in other pediatric studies [45]. Where possible, analyses were adjusted for confounders, as well as the multiple comparisons made. However, the rarity of pediatric MS combined with lack of prior work meant that relatively small groups of individuals were enrolled into these pilot studies, limiting ability to interrogate the gut microbiota composition below broad taxonomic groupings (e.g., phylum-level when assessing relapse risk [17]). The small sample size also limited sophistication of covariate adjustment. However, these studies represent important first steps into understanding the role of the gut microbiota in complex conditions such as MS [16-18].

\section{Future Perspectives}

As with all observational studies, causation cannot be inferred. However, the associations found to date suggest a relationship may exist between the gut microbiota and the host's immune profile, as well as the gut microbiota and disease risk and activity in MS. The gut microbiota may provide a modifiable target in order to positively influence these disease-related characteristics and outcomes in MS. The current work predominantly refers to the taxonomy of gut bacteria (and some Archaea); future lines of inquiry will include the fungiome, virome, and the functional ability of these diverse microbes. The capacity for these and other more sophisticated assessments and analyses of the gut microbiota will improve as sequencing methods, bioinformatics, and computational capacity advances. Ultimately, a better understanding of the relationships between the gut microbiota and human host could be key in facilitating our understanding of complex conditions such as MS.

Two collaborative efforts are underway to validate and extend these findings in much larger cohorts of children involving both the US Network of Pediatric MS Centers and the Canadian Pediatric Demyelinating Disease Study team (lead by Dr. Banwell). Both the US National MS Society (Principal Investigator: Waubant) and MS Society of Canada's MS Scientific and Research Foundation (Principal Investigator: Tremlett) have dedicated resources, allowing the establishment of a stool biobank including children with earlyonset pediatric MS and related demyelinating conditions along with healthy controls ("From bugs to brains: the gut microbiome in pediatric MS": see Acknowledgments). This endeavour will enable access to larger sample sizes, increased study power, and validation and replication of findings, and is to open to collaboration (contact the study authors: Drs. Tremlett or Waubant).

Acknowledgments "From bugs to brains: the gut microbiome in pediatric MS". Funding body: The Multiple Sclerosis Scientific and Research Foundation. Principal Investigator (PI): Helen Tremlett (University of British Columbia). Co-PIs: Gary Van Domselaar, Charles Bernstein, Morag Graham (University of Manitoba and the National Microbiology Laboratory); Emmanuelle Waubant (University of California, San Francisco). US National Multiple Sclerosis Society grant RG4861A3. PI: Emmanuelle Waubant (University of California, San Francisco), and the US Pediatric MS Network.

Required Author Forms Disclosure forms provided by the authors are available with the online version of this article.

\section{Compliance with ethical standards}

Conflicts of interest HT-none in relation to this work. HT is the Canada Research Chair in Neuroepidemiology and Multiple Sclerosis. She currently receives research support from the National Multiple Sclerosis Society, the Canadian Institutes of Health Research, the Multiple Sclerosis Society of Canada and the Multiple Sclerosis Scientific Research Foundation. In addition, in the last 5 years she has received research support from the Multiple Sclerosis Society of Canada (Don Paty Career Development Award); the Michael Smith Foundation for Health Research (Scholar Award), and the UK MS Trust; speaker honoraria and/or travel expenses to attend conferences from the Consortium of MS Centres (2013), the National MS Society (2012, 2014, 2016), ECTRIMS $(2012,2013,2014,2015,2016)$, the Chesapeake Health Education Program, US Veterans Affairs (2012), Novartis Canada (2012), Biogen Idec (2014), amd American Academy of Neurology $(2013,2014,2015,2016)$. All speaker honoraria are either declined or donated to an MS charity or to an unrestricted grant for use by her research group.

EW-none in relation to this work. Dr. Waubant is funded by the National Institutes of Health, the National MS Society, and the Race to Erase MS.

\section{References}

1. Banwell B, Bar-Or A, Arnold DL, et al. Clinical, environmental, and genetic determinants of multiple sclerosis in children with acute demyelination: a prospective national cohort study. Lancet Neurol 2011;10:436-445.

2. Waldman A, Ghezzi A, Bar-Or A, et al. Multiple sclerosis in children: an update on clinical diagnosis, therapeutic strategies, and research. Lancet Neurol 2014;13:936-948. 
3. Gianfrancesco MA, Stridh P, Rhead B, et al. Evidence for a causal relationship between low vitamin $\mathrm{D}$, high $\mathrm{BMI}$, and pediatric-onset MS. Neurology 2017;88:1623-1629.

4. Waubant E, Ponsonby A-L, Pugliatti M, et al. Environmental and genetic factors in pediatric inflammatory demyelinating diseases. Neurology 2016;87:S20-S27.

5. McKay KA, Jahanfar S, Duggan T, Tkachuk S, Tremlett H. Factors associated with onset, relapses or progression in multiple sclerosis: a systematic review. Neurotoxicology 2017; 61:189-212.

6. Tremlett H, Zhao Y, Rieckmann P, Hutchinson M. New perspectives in the natural history of multiple sclerosis. Neurology 2010;74:2004-2015.

7. Lee YK, Menezes JS, Umesaki Y, Mazmanian SK. Proinflammatory T-cell responses to gut microbiota promote experimental autoimmune encephalomyelitis. Proc Natl Acad Sci U S A 2011;108:4615-4622.

8. Berer K, Mues M, Koutrolos M, et al. Commensal microbiota and myelin autoantigen cooperate to trigger autoimmune demyelination. Nature 2011;479:538-541.

9. Tremlett H, Bauer KC, Appel-Cresswell S, Finlay BB, Waubant E. The gut microbiome in human neurological disease: a review. Ann Neurol 2017;81:369-382.

10. Cantarel BL, Waubant E, Chehoud C, et al. Gut microbiota in multiple sclerosis: possible influence of immunomodulators. J Investig Med 2015;63:729-734.

11. Jangi S, Gandhi R, Cox LM, et al. Alterations of the human gut microbiome in multiple sclerosis. Nat Commun 2016;7:12015.

12. Miyake S, Kim S, Suda W, et al. Dysbiosis in the gut microbiota of patients with multiple sclerosis, with a striking depletion of species belonging to clostridia XIVa and IV clusters. PLoS ONE 2015;10: e0137429.

13. Rumah KR, Linden J, Fischetti VA, Vartanian T. Isolation of Clostridium perfringens type B in an individual at first clinical presentation of multiple sclerosis provides clues for environmental triggers of the disease. PLoS ONE 2013;8:e76359.

14. Chen J, Chia N, Kalari KR, et al. Multiple sclerosis patients have a distinct gut microbiota compared to healthy controls. Sci Rep 2016;6:28484.

15. Wijnands JMA, Kingwell E, Zhu F, et al. Health-care use before a first demyelinating event suggestive of a multiple sclerosis prodrome: a matched cohort study. Lancet Neurol 2017;16:445-451.

16. Tremlett H, Fadrosh D, Faruqi A, et al. Gut microbiota in early pediatric multiple sclerosis: a case-control study. Eur J Neurol 2016;23:1308-1321.

17. Tremlett H, Fadrosh DW, Faruqi AA, et al. Gut microbiota composition and relapse risk in pediatric MS: a pilot study. J Neurol Sci 2016;363:153-157.

18. Tremlett H, Fadrosh DW, Faruqi AA et al. Associations between the gut microbiota and host immune markers in pediatric multiple sclerosis and controls. BMC Neurol 2016;16:182.

19. Polman CH, Reingold SC, Banwell B, et al. Diagnostic criteria for multiple sclerosis: 2010 revisions to the McDonald criteria. Ann Neurol 2011;69:292-302.

20. Goodrich JK, Di Rienzi SC, Poole AC, et al. Conducting a microbiome study. Cell 2014;158:250-262.

21. Nagalingam NA, Lynch SV. Role of the microbiota in inflammatory bowel diseases. Inflamm Bowel Dis 2012;18:968-984.

22. Ley RE, Turnbaugh PJ, Klein S, Gordon JI. Microbial ecology: human gut microbes associated with obesity. Nature 2006;444: 1022-1023.

23. International Multiple Sclerosis Genetics Consortium, Beecham AH, Patsopoulos NA, et al. Analysis of immune-related loci identifies 48 new susceptibility variants for multiple sclerosis. Nat Genet 2013;45:1353-1360.
24. Graves JS, Chitnis T, Weinstock-Guttman B, et al. Maternal and perinatal exposures are associated with risk for pediatric-onset multiple sclerosis. Pediatrics 2017;139:e20162838.

25. Loubinoux J, Bronowicki JP, Pereira IA, Mougenel JL, Faou AE. Sulfate-reducing bacteria in human feces and their association with inflammatory bowel diseases. FEMS Microbiol Ecol 2002;40:107112.

26. Rowan F, Docherty NG, Murphy M, et al. Desulfovibrio bacterial species are increased in ulcerative colitis. Dis Colon Rectum 2010;53:1530-1536.

27. Di Cagno R, De Angelis M, De Pasquale I, et al. Duodenal and faecal microbiota of celiac children: molecular, phenotype and metabolome characterization. BMC Microbiol 2011;11:219.

28. Nistal E, Caminero A, Vivas S, et al. Differences in faecal bacteria populations and faecal bacteria metabolism in healthy adults and celiac disease patients. Biochimie 2012;94:1724-1729.

29. Benjamin JL, Hedin CR, Koutsoumpas A, et al. Smokers with active Crohn's disease have a clinically relevant dysbiosis of the gastrointestinal microbiota. Inflamm Bowel Dis 2012;18:10921100.

30. Wang W, Chen L, Zhou R, et al. Increased Proportions of Bifidobacterium and the Lactobacillus group and loss of butyrateproducing bacteria in inflammatory bowel disease. J Clin Microbiol 2014;52:398-406.

31. Segain J, de la Bletiere DR, Bourreille A, et al. Butyrate inhibits inflammatory responses through $\mathrm{NF} \kappa \mathrm{B}$ inhibition: implications for Crohn's disease. Gut 2000;47:397-403.

32. Langille MG, Zaneveld J, Caporaso JG, et al. Predictive functional profiling of microbial communities using 16S rRNA marker gene sequences. Nat Biotechnol 2013;31:814-821.

33. Carvalho AN, Lim JL, Nijland PG, Witte ME, Van Horssen J. Glutathione in multiple sclerosis: more than just an antioxidant? Mult Scler 2014;20:1425-1431.

34. Julio-Pieper M, Flor PJ, Dinan TG, Cryan JF. Exciting times beyond the brain: metabotropic glutamate receptors in peripheral and non-neural tissues. Pharmacol Rev 2011;63:35-58.

35. Yong VW. Differential mechanisms of action of interferon- $\beta$ and glatiramer acetate in MS. Neurology 2002;59:802-808.

36. Tremlett H, Waubant E. The multiple sclerosis microbiome? Ann Transl Med 2017;5:53.

37. Rooks MG, Garrett WS. Gut microbiota, metabolites and host immunity. Nat Rev Immunol 2016;16:341-352.

38. Honda K, Littman DR. The microbiota in adaptive immune homeostasis and disease. Nature 2016;535:75-84.

39. Steinman L. Immunology of relapse and remission in multiple sclerosis. Annu Rev Immunol 2014;32:257-281.

40. Jones AP, Kermode AG, Lucas RM et al. Circulating immune cells in multiple sclerosis. Clin Exp Immunol 2017;187:193-203.

41. Mielcarz DW, Kasper LH. The gut microbiome in multiple sclerosis. Curr Treat Options Neurol 2015;17:344.

42. Maynard CL, Elson CO, Hatton RD, Weaver CT. Reciprocal interactions of the intestinal microbiota and immune system. Nature 2012;489:231-241.

43. Ochoa-Repáraz J, Mielcarz DW, Ditrio LE, et al. Central nervous system demyelinating disease protection by the human commensal Bacteroides fragilis depends on polysaccharide A expression. J Immunol 2010;185:4101-4108.

44. Wacholder S, Silverman DT, McLaughlin JK, Mandel JS. Selection of controls in case-control studies: II. Types of controls Am J Epidemiol 1992;135:1029-1041.

45. Schendel DE, Parner E. Sibling comparisons and confounding in autism epidemiological studies. JAMA Psychiat 2016;73:302-303. 